\section{USO DE RECURSOS DE INFORMACIÓN DISPONIBLES EN INTERNET: LIMITACIONES Y RECOMENDACIONES}

\author{
USE OF INFORMATION RESOURCES \\ AVAILABLE ON THE INTERNET: LIMITATIONS \\ AND RECOMMENDATIONS
}

Emily Orihuela-Onofre ${ }^{1, a}$, Eduardo Garibay-Yalan ${ }^{1, a}$

Sr. Editor. Leímos con mucho interés el artículo de Mejía C. et al. donde, de manera descriptiva, muestra datos relevantes sobre el uso de fuentes de información en médicos recién graduados de Lima ${ }^{(1)}$. Sin embargo, creemos necesario profundizar algunos aspectos del estudio que fueron soslayados en las discusiones y recomendaciones propuestas por los autores.

Actualmente el estudiante de medicina tiene mayor acceso a información por Internet, a la que acude con fines científico-académicos ${ }^{(2)}$, para esto hace uso de recursos disponibles como Pubmed, SciELO, HINARI, LILACS, entre otros; en este estudio se encontró una baja frecuencia del uso de estos recursos y consideran el dominio del inglés como posible factor limitante, respecto a esto, los autores no plasmaron de forma correcta la información brindada por su fuente bibliográfica, en donde llegaron a demostrar que el bajo dominio de este idioma sí es un factor limitante, debido a que la mayor cantidad de información con el mejor nivel científico se encuentra en inglés $y$, por otro lado, a la existencia de un gran porcentaje de estudiantes con un escaso dominio y comprensión de artículos en este idioma ${ }^{(2,3)}$.

Otra posible causa de la baja frecuencia de uso de recursos de información virtual planteada en el estudio refiere que los alumnos no encuentran utilidad o no ven la necesidad de buscar información por estos medios y aunque no fue evaluado, los autores no tomaron en cuenta artículos previos sobre el tema, pues en un estudio realizado en la Universidad Nacional Mayor de San Marcos concluyeron que durante los primeros años de la carrera los estudiantes de Medicina buscan información, principalmente en libros de ciencias

\footnotetext{
Sociedad Científica San Fernando, Facultad de Medicina, Universidad Nacional Mayor de San Marcos. Lima, Perú.

a Estudiante de Medicina.

Recibido: 23-01-15 Aprobado: 28-01-15
}

Citar como: Orihuela-Onofre E, Garibay-Yalan E. Uso de recursos de información disponibles en internet: limitaciones y recomendaciones [carta]. Rev Peru Med Exp Salud Publica. 2015;32(1):200-1. básicas, y hacen poco uso de las bases de datos disponibles en Internet, pero es a partir del contacto con los pacientes cuando surge la necesidad de investigar $y$ el uso de los recursos de fuentes de información tiende a aumentar en frecuencia de primero a sexto año ${ }^{(2)}$, esto podría ser explicado por el grado de madurez clínica que los alumnos adquieren con el tiempo, influyendo en la percepción sobre la importancia de una efectiva y eficiente búsqueda de literatura médica (4).

Al final del estudio recomiendan evaluar las diversas estrategias de capacitación dirigidas a usar eficientemente los diversos recursos de información relacionados con salud, y de manera continua en estudiantes de medicina y profesionales de la salud, no obstante, no consideraron publicaciones que evalúan la efectividad de diversas estrategias de capacitación en búsqueda bibliográfica, como el trabajo de Just quien evaluó 15 trabajos publicados entre 1998-2011, que contaron con una descripción de su intervención, un resumen de la prueba utilizada para medir el aprendizaje y los resultados de la medición; en todos ellos se encontraron mejoras, tanto en la formulación de la pregunta de investigación, en la construcción de la estrategia de búsqueda bibliográfica, en la selección de artículos y en el uso de los recursos informáticos; sin embargo, a pesar de que la mayoría de estos estudios evidenciaron que las estrategias de capacitación son eficaces, algunos mostraron debilidades en sus diseños, porque los métodos utilizados para la evaluación de las diferentes habilidades desarrolladas en las capacitaciones no fueron rigurosamente elaborados ${ }^{(5)}$.

Ante lo expuesto, recomendamos investigar más sobre el uso y dominio del idioma inglés en las etapas preclínica y clínica para medir impacto que tiene esta limitante en la obtención de información y aprendizaje en los años académicos. Con relación a la evaluación de las capacitaciones, se debe elaborar diseños mejor estructurados con el fin de verificar la efectividad de estrategias de capacitación en búsqueda de información en bases de datos, y que se considere la retención de estas habilidades en el tiempo.

Conflictos de interés: los autores declaran no tener conflictos de interés.

Fuentes de financiamiento: autofinanciado.

\section{REFERENCIAS BIBLIOGRÁFICAS}

1. Mejia CR, Caceres OJ, Vera CA, Nizama-Vía A, Curioso WH, Mayta-Tristán P. Uso de fuentes de información en médicos recién graduados de Lima. Rev Peru Med Exp Salud Pública. 2014;31(4):716-20. 
2. Angulo-Bazán Y, Borja-García R, Alegre-Tuesta T, AlvaGutierrez C, Gutiérrez-Zegarra G. Gestión de la información cientifica en estudiantes de medicina de una universidad pública peruana. CIMEL. 2010;15(1):23-7.

3. Veloz-Martínez MG, Almanza-Velasco E, Uribe-Ravell JA, Libiend-Díaz González L, Quintana-Romero V, Alanís-López P. Uso de tecnologías en información y comunicación por médicos residentes de ginecología y obstetricia. Inv Ed Med. 2012;1(4):183-9.

4. Llic D, Tepper K, Misso M. Teaching evidence-based medicine literature searching skills to medical students during the clinical years: a randomized controlled trial. J Med Libr Assoc. 2012 Jul;100(3):190-6. doi: 10.3163/1536-5050.100.3.009.

5. Just ML. Is literature search training for medical students and residents effective? a literature review. J Med Libr Assoc. 2012 Oct;100(4):270-6. doi: 10.3163/1536-5050.100.4.008.

Correspondencia: Emily Elizabeth Orihuela Onofre Dirección: Av. General Vivanco 539 - Interior C2, Pueblo Libre, Lima 21, Perú.

Teléfono: 964552772

Correo electrónico: xemilyelix@gmail.com

\section{COMENTARIOS AL ARTÍCULO: GASOMETRÍA ARTERIAL EN ADULTOS CLIINICAMENTE SANOS A 3350 METROS DE ALTITUD}

\author{
COMMENTS TO THE ARTICLE: ARTERIAL \\ BLOOD GASES IN CLINICALLY HEALTHY \\ ADULTS AT 3,350 METERS ABOVE SEA LEVEL \\ Virgilio E. Failoc-Rojas ${ }^{1,2, a}$
}

Sr. Editor. He leído con interés el artículo publicado por Pereira-Victorio CJ et al. (1) en el que da un enfoque de los parámetros gasométricos arteriales en población adulta sana a $\mathbf{3 3 5 0}$ metros de altitud, explicando el cambio y adaptación fisiológica en el poblador altoandino.

En el artículo, los aspectos éticos como el consentimiento informado y los conflictos de interés son necesarios y sí están manifestados, sin

\footnotetext{
1 Universidad Nacional Pedro Ruiz Gallo. Lambayeque, Perú

Sociedad Científica Estudiantes de Medicina (SOCIEM-UNPRG). Lambayeque, Perú.

Estudiante de Medicina.

Recibido: 26-01-15 Aprobado: 28-01-15
}

Citar como: Failoc-Rojas VE. Comentarios al artículo: gasometría arterial en adultos clínicamente sanos a 3350 metros de altitud [carta]. Rev Peru Med Exp Salud Publica. 2015;32(1):201-2. embargo, no mencionan si el artículo fue aprobado por un comité de ética institucional, siendo un estudio que demandó investigaciones invasivas en seres humanos. Es importante que las investigaciones científicas tengan aprobación de un comité de ética (2) y los editores de revistas científicas deben solicitar se declaren los aspectos mencionados ${ }^{(3)}$. Esto es muy significativo, como recomendación para poder alcanzar mejores estándares y tener un mayor impacto de las publicaciones científicas peruanas a nivel internacional.

En cuanto a la evaluación para los criterios de inclusión, en el resumen mencionan que fue evaluado por dos médicos internistas, mientras que en la sección Materiales y Métodos refieren que fue evaluado por tres médicos internistas. Es necesario aclarar este punto, pues puede influenciar en la selección de pacientes; además, la selección muestral fue limitada y no probabilística. Se debe rescatar que el estudio da una visión de los parámetros gasométricos de las personas que viven en la altura, sin embargo, no se pueden dar recomendaciones para extrapolar los resultados.

Es importante resaltar que los resultados que muestra el artículo pueden haber sido diferentes de los estudios que contrastan, porque los criterios de selección no están bien establecidos. También considero que debe aclarar si se excluyó a pacientes embarazadas, pacientes con uso excesivo del alcohol, y la realización de pruebas de función renal, pues estos parámetros pueden alterar el equilibrio ácido-base ${ }^{(4)}$.

Los criterios de selección son muy importantes, pues servirán para evitar sesgos de selección $y$, sobre todo, para obtener resultados más confiables, lo cual puede considerar el porqué de las diferencias con los estudios contrastados.

Conflictos de Interés: el autor declara no tener conflicto de interés.

Fuentes de financiamiento: autofinanciado.

\section{REFERENCIAS BIBLIOGRÁFICAS}

1. Pereira-Victorio CJ, Huamanquispe-Quintana J, CasteloTamayo LE. Gasometría arterial en adultos clínicamente sanos a 3350 metros de altitud. Rev Peru Med Exp Salud Publica. 2014;31(3):473-9.

2. Rossato N. Aspectos éticos de las publicaciones. Arch Argent Pediatr. 2014;112(6):490-1. 